\title{
Effect of Helicobacter pylori Treatment on Long-term Mortality in Patients with Hypertension
}

\author{
Young-II Kim ${ }^{1}$, Young Ae Kim², Jang Won Lee, ${ }^{3}$ Hak Jin Kim ${ }^{4}$, Su-Hyun Kim${ }^{5}$, Sang Gyun Kim ${ }^{6}$, Jin II Kim , Jae J. Kim , and II \\ Ju Choi ${ }^{1}$ \\ ${ }^{1}$ Center for Gastric Cancer and ${ }^{2}$ Cancer Survivorship Branch, National Cancer Control Institute, National Cancer Center, Goyang, ${ }^{3}$ College \\ of Korean Medicine, Dongguk University, Gyeongju, ${ }^{4}$ Department of Cardiology, Center for Clinical Specialty, National Cancer Center, \\ ${ }^{5}$ Department of Neurology, Research Institute and Hospital, National Cancer Center, Goyang, ${ }^{6}$ Department of Internal Medicine and Liver \\ Research Institute, Seoul National University College of Medicine, ${ }^{7}$ Department of Internal Medicine, Yeouido St. Mary's Hospital, College \\ of Medicine, The Catholic University of Korea, and ${ }^{8}$ Department of Medicine, Samsung Medical Center, Sungkyunkwan University School of \\ Medicine, Seoul, Korea
}

Background/Aims: A meta-analysis of randomized trials performed in healthy asymptomatic individuals suggested that overall mortality may increase after Helicobacter pylori eradication despite a significant decrease in the gastric cancer incidence and mortality rates. This retrospective population-based cohort study investigated if $H$. pylori treatment is associated with an increase in overall mortality in patients with hypertension. Methods: From the database of the Korean National Health Insurance Sample Cohort, we selected 198,487 patients treated for hypertension between 2002 and 2010. Those who received $H$. pylori treatment $(H$. pylori treatment cohort, 5,541 patients) were matched to those who did not (nontreatment cohort, 11,082 patients) at the ratio of 1 to 2 . The primary outcome was the risk of overall mortality. The secondary outcomes were the risks of mortality due to cardiovascular disease, cerebrovascular disease, and cancer. The outcomes were evaluated from 6 months after $H$. pylori treatment to December 2013. A Cox proportional hazard model was used to estimate the hazard ratios (HRs). Results: During a median follow-up period of 4.8 years, death from any cause was reported in $4.1 \%$ of the patients in the $H$. pylori treatment cohort and $5.5 \%$ of the patients in the nontreatment cohort. The adjusted HR (aHR) for overall mortality in the $H$. pylori treatment cohort was 0.70 (95\% confidence interval [Cl], 0.60 to $0.82 ; \mathrm{p}<0.001)$. With regard to cause-specific mortality, compared with the nontreatment cohort, the $H$. pylori treatment cohort had a lower risk of mortality due to cerebrovascular disease (aHR, 0.46 ; $95 \% \mathrm{Cl}, 0.26$ to $0.81 ; p=0.007)$. The risks of mortality due to cancer and cardiovascular disease were not different between the cohorts. Conclusions: $H$. pylori treatment is not associated with an increase in overall mortality in patients treated for hypertension. (Gut Liver 2020;14:47-56)

Key Words: Helicobacter pylori; Mortality; Hypertension

\section{INTRODUCTION}

Helicobacter pylori infection is an important risk factor for gastric cancer and is categorized as a group I carcinogen. ${ }^{1} \mathrm{~A}$ meta-analysis of six randomized trials performed in asymptomatic individuals reported that $H$. pylori eradication reduced gastric cancer incidence (relative risk, 0.66) and mortality (relative risk, 0.67). ${ }^{2}$ This meta-analysis, however, showed an approximately $9 \%$ nonsignificant increase in overall mortality after $H$. pylori treatment. ${ }^{2}$ Although the detailed causes of death were not available from these studies, cardiovascular, cerebrovascular, or other cancer mortalities could be the major causes of death according to worldwide statistics. ${ }^{3-5}$

H. pylori treatment regimens consist of a combination of broad-spectrum antibiotics, including amoxicillin, clarithromycin, tetracycline, or metronidazole. ${ }^{6}$ Among those antibiotics, concerns about a link between clarithromycin use and cardiovascular mortality have been reported. A randomized trial performed in patients with stable coronary heart disease showed that short-term clarithromycin treatment (2 weeks) significantly increased cardiovascular mortality during a 3-year follow-up and overall mortality during a 6-year follow-up. ${ }^{7,8}$ In a metaanalysis including 33 studies investigating the associations

Correspondence to: Il Ju Choi

Center for Gastric Cancer, National Cancer Center, 323 Ilsan-ro, Ilsandong-gu, Goyang 10408, Korea

Tel: +82-31-920-2282, Fax: +82-31-920-2799, E-mail: cij1224@ncc.re.kr

Received on November 21, 2018. Revised on January 17, 2019. Accepted on January 26, 2019. Published online July $24,2019$. pISSN 1976-2283 eISSN 2005-1212 https://doi.org/10.5009/gnl18510

(a) This is an Open Access article distributed under the terms of the Creative Commons Attribution Non-Commercial License (http://creativecommons.org/licenses/by-nc/4.0) which permits unrestricted non-commercial use, distribution, and reproduction in any medium, provided the original work is properly cited. 
between macrolide antibiotics and cardiovascular risk, clarithromycin was significantly associated with increased cardiovascular disease mortality and overall mortality. ${ }^{9}$ Antibiotic treatment also causes disruption of gut microbiota (dysbiosis), which could affect various steps in the process of carcinogenesis (cancer initiation, progression, and dissemination) or disrupt the efficacy of anti-cancer treatments. ${ }^{10,11}$ A recent nested case-control study using a UK medical records database reported a possible association between repeated macrolide antibiotics use and increased risk of various types of cancers. ${ }^{12}$

Hypertension is an important risk factor for death from cardiovascular and cerebrovascular diseases. ${ }^{13}$ Thus, we selected patients with hypertension from the database of the Korean National Health Insurance population-based sample cohort. In the present study, we investigated if $H$. pylori treatment in patients with hypertension is associated with overall mortality or with mortality from major causes.

\section{MATERIALS AND METHODS}

\section{Study design and data source}

This study is a retrospective population-based cohort study using the Korean National Health Insurance Service-National Sample Cohort database. This study was approved by the Institutional Review Board of the National Cancer Center, Korea (IRB number: NCC2016-0043) and informed consent requirements for the individuals in the database were waived.

We selected a study population from the database, ${ }^{14}$ which is a population-based sample cohort established to provide representative and useful health insurance and health examination data to public health researchers and policymakers. To summarize this cohort, approximately one million subjects $(2.2 \%$ of the total eligible population) were randomly sampled from the 2002 Korean National Health Insurance database and followed up until 2013. This database has been well-validated and increasingly used for epidemiological and health policy studies for general Korean populations. ${ }^{15,16}$

The database includes participants' insurance eligibility, medical treatments, medical care institutions, and general health examinations data. In the insurance eligibility database, variables include the participant's identity and socioeconomic information (gender, residential area, type of health insurance, level of income, birth, and death). The medical treatment database contains the participant's medical treatment bills, bill details, details of disease, and prescriptions. The general health examination database includes major health exam results and information about lifestyles and behaviors from questionnaires. ${ }^{14}$ To identify subjects' diagnosis and cause of mortality, disease codes from the International Classification of Diseases (ICD) 10th revision were used. ${ }^{17}$

\section{Study population}

We selected patients with hypertension from the database. Patients who were aged $\geq 20$ years, diagnosed with hypertension (ICD-10 code, I10) between 2002 and 2010, and had been prescribed at least a one anti-hypertensive drug were included in our study as patients with hypertension.

The prescription of $H$. pylori treatment regimens-protonpump inhibitor (PPI)-clarithromycin-containing triple therapy (PPIs, clarithromycin, and amoxicillin) or bismuth-containing quadruple therapy (bismuth, PPIs, metronidazole, and tetracycline)-was examined using drug codes. In Korea, those treatment regimens were approved as the first-line and the secondline treatment, respectively, by the guidelines and National Health Insurance. ${ }^{18}$ The $H$. pylori treatment cohort included patients who were prescribed $H$. pylori treatment regimens after a hypertension diagnosis up to June 2013.

Patients in the $H$. pylori treatment cohort were matched with those in the nontreatment cohort at a ratio of 1:2 using the simple random sampling without replacement method. Variables of age group, sex, hypertension diagnosis date (month and year) and Charlson comorbidity index (CCI) score $^{19}$ at the time of hypertension diagnosis were included for matching.

Of the patients in the H. pylori treatment cohort, patients who died from any cause or those who were diagnosed with any cancers, cardiovascular diseases, or cerebrovascular diseases before or within the 6 months after $H$. pylori treatments were excluded. In the nontreatment cohort, we also excluded patients who died due to any causes or those who were diagnosed with any cancers, cardiovascular diseases, or cerebrovascular diseases before or within 6 months after the corresponding date for $H$. pylori treatment prescription of the matched case.

\section{Observation periods}

To define the starting time of observation periods for study outcomes in the nontreatment cohort, the corresponding date of $H$. pylori treatment prescription in the treatment cohort was assigned to each patient in the nontreatment cohort after matching. Thus, the observation period was started from the date of $H$. pylori treatment prescription in the treatment cohort and the corresponding date in the nontreatment cohort. These observation periods were continued until December 2013 (Supplementary Fig. 1).

\section{Study outcomes}

The primary outcome was overall mortality, defined as a death from any cause occurring from 6 months after the starting time of the observation period. Secondary outcomes included cancer-specific mortality, cardiovascular disease-specific mortality, and cerebrovascular-specific mortality occurring from 6 months after the starting time of the observation period. The disease codes describing the cause of death in the database 
were identified to define disease-specific mortalities, and disease codes were ICD-10 code C00-97 for cancers, I20-I25 for cardiovascular diseases, and I60-I66 for cerebrovascular diseases (Supplementary Table 1).

\section{Statistical analysis}

Descriptive analyses were performed to compare patients' baseline characteristics between the $H$. pylori treatment cohort and nontreatment cohort. The Student t-test and chi-square test were used to compare differences between cohorts.

Kaplan-Meier curves with log-rank test were performed for the comparisons of overall mortality and cause-specific mortalities between $H$. pylori treatment and nontreatment cohorts. Univariate and multivariate Cox proportional hazard models were used to estimate hazard ratios (HR) with 95\% confidential interval (CI) for mortalities and incidences of cancers, cardiovascular, and cerebrovascular diseases according to the H. pylori treatment. Covariates included for the multivariate analysis were age group, sex, economic status, residential area, CCI scores, and the use of aspirin and statin. All statistical analyses were performed using SAS version 9.4 (SAS Institute, Cary, NC, USA). p-values of $<0.05$ were considered statistically significant.

\section{RESULTS}

\section{Baseline characteristics of the study population}

The study flow is presented in Fig. 1. A total of 198,487 patients were diagnosed with hypertension and prescribed hypertension medication at least once. Of these, 9,552 patients were prescribed $H$. pylori treatment regimens between the hypertension diagnosis and June 2013. After excluding 3,661 patients who died or were diagnosed with cancer, cardiovascular, or cerebrovascular diseases before or within 6 months of $H$. pylori treatment to exclude preexisting conditions, 5,891 patients were included for case-control matching. A total of 5,541 patients in the $H$. pylori treatment cohort and 11,082 matched patients in the nontreatment cohort were included for the final analyses.

In the H. pylori treatment cohort, 96.4\% of patients were prescribed with PPI-clarithromycin-containing triple therapy (Table 1). Baseline characteristics were similar between the two co-

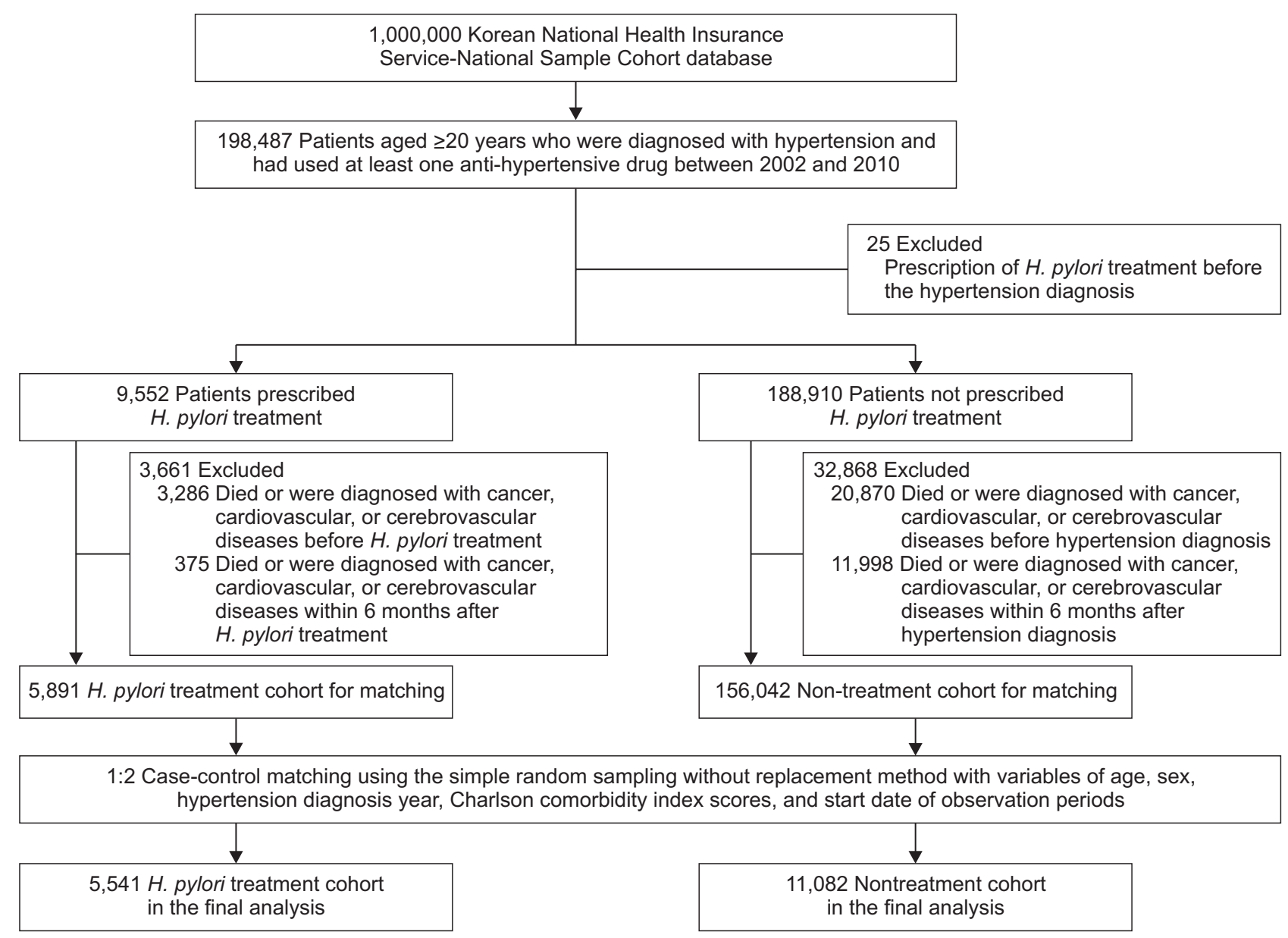

Fig. 1. Study flowchart. Patients with hypertension who had used at least one anti-hypertensive drug were sorted into the Helicobacter pylori treatment cohort or nontreatment cohort. Patients in the H. pylori treatment cohort were matched with those in the nontreatment cohort at the ratio of 1 to 2 by using simple random sampling without replacement. 
50 Gut and Liver, Vol. 14, No. 1, January 2020

Table 1. Baseline Characteristics of the Study Population

\begin{tabular}{|c|c|c|c|}
\hline Characteristic & H. pylori treatment cohort $(\mathrm{n}=5,541)$ & Nontreatment cohort $(\mathrm{n}=11,082)$ & p-value \\
\hline Age, yr & & & 1.00 \\
\hline $20-29$ & $41(0.7)$ & $82(0.7)$ & \\
\hline $30-39$ & $424(7.7)$ & $848(7.7)$ & \\
\hline $40-49$ & $1,526(27.5)$ & $3,052(27.5)$ & \\
\hline $50-59$ & $1,968(35.5)$ & $3,936(35.5)$ & \\
\hline $60-69$ & $1,297(23.4)$ & $2,594(23.4)$ & \\
\hline $70-79$ & $268(4.8)$ & $536(4.8)$ & \\
\hline$\geq 80$ & $17(0.3)$ & $34(0.3)$ & \\
\hline Sex & & & 1.00 \\
\hline Male & $3,059(55.2)$ & $6,118(55.2)$ & \\
\hline Female & $2,482(44.8)$ & $4,964(44.8)$ & \\
\hline Residential area & & & $<0.001$ \\
\hline Metropolitan & $2,745(49.5)$ & $5,180(46.7)$ & \\
\hline Small city or rural & $2,796(50.5)$ & $5,902(53.3)$ & \\
\hline Economic status level, \% & & & $<0.001$ \\
\hline $0-50$ & $2,060(37.2)$ & $4,452(40.2)$ & \\
\hline $51-100$ & $3,481(62.8)$ & 6,630 (59.8) & \\
\hline Smoking & & & $<0.001$ \\
\hline Never smoker & $2,574(46.5)$ & $4,652(42.0)$ & \\
\hline Ex-smoker & $520(9.4)$ & $844(7.6)$ & \\
\hline Current smoker & $1,045(18.9)$ & $1,520(13.7)$ & \\
\hline Missing & $1,402(25.3)$ & $4,066(36.7)$ & \\
\hline Alcohol drinking & & & 0.863 \\
\hline Never drinking & $2,383(43.0)$ & $4,002(36.1)$ & \\
\hline $1-2$ times/wk & $1,245(22.5)$ & $2,127(19.2)$ & \\
\hline 3-4 times/wk & $414(7.5)$ & $733(6.6)$ & \\
\hline 5-7 times/wk & $245(4.4)$ & 425 (3.8) & \\
\hline Missing & $1,254(22.6)$ & $3,795(34.2)$ & \\
\hline Body mass index, $\mathrm{kg} / \mathrm{m}^{2}$ & & & 0.204 \\
\hline$\leq 18.5$ & $46(0.8)$ & $95(0.9)$ & \\
\hline $18.6-22.9$ & $1,065(19.2)$ & $1,845(16.6)$ & \\
\hline $23.0-24.9$ & $1,208(21.8)$ & $1,934(17.5)$ & \\
\hline$\geq 25.0$ & $2,051(37.0)$ & $3,556(32.1)$ & \\
\hline Missing & $1,171(21.1)$ & $3,652(33.0)$ & \\
\hline CCI score at the time of hypertension diagnosis & & & 1.00 \\
\hline 0 & $2,955(53.3)$ & $5,910(53.3)$ & \\
\hline 1 & $866(15.6)$ & $1,732(15.6)$ & \\
\hline$\geq 2$ & $1,720(31.0)$ & $3,440(31.0)$ & \\
\hline Aspirin use* & & & 0.21 \\
\hline No & $4,386(79.2)$ & $8,864(80.0)$ & \\
\hline Yes & $1,155(20,8)$ & $2,218(20.0)$ & \\
\hline Statin use $\mathrm{e}^{*}$ & & & $<0.001$ \\
\hline No & $4,513(81.4)$ & $9,434(85.1)$ & \\
\hline Yes & $1,028(18.6)$ & $1,648(14.9)$ & \\
\hline \multicolumn{4}{|l|}{ H. pylori treatment regimen } \\
\hline PPI-clarithromycin containing triple therapy & $5,342(96.4)$ & - & \\
\hline Bismuth-containing quadruple therapy & $72(1.3)$ & - & \\
\hline Both & $127(2.3)$ & - & \\
\hline Follow-up period, yr & $4.8(2.6-7.4)$ & $4.8(2.6-7.3)$ & 0.216 \\
\hline
\end{tabular}

Data are presented number (\%) or median (interquartile range).

H. pylori, Helicobacter pylori; CCI, Charlson comorbidity index; PPI, proton-pump inhibitor.

*Patients who used aspirin and statin were defined as those who were prescribed the drugs for at least 30 days. 
horts, except that patients in the $H$. pylori treatment cohort had a higher proportion of residency in metropolitan areas (49.5\% vs $46.7 \%, \mathrm{p}<0.001)$ and statin use $(18.6 \%$ vs $14.9 \%, \mathrm{p}<0.001)$ than those in the nontreatment cohort.

\section{Risk of overall mortality after $\boldsymbol{H}$. pylori treatment}

During the observation periods until December 2013 (median, 4.8 years; interquartile range, 2.6 to 7.3 years; 85,078 personyears), death occurred in 837 patients (5.0\%); 229 patients (4.1\%) in the H. pylori treatment cohort and 608 (5.5\%) in the nontreatment cohort. Overall survival in the $H$. pylori treatment cohort was significantly higher when compared with that in the nontreatment cohort ( $<<0.001$ by the log-rank test) (Fig. 2). The 5-year overall survival rates were $96.7 \%$ in the $H$. pylori treatment cohort and 95.3\% in the nontreatment cohort. A multivariate analysis also showed a significantly reduced risk for overall mortality in the $H$. pylori treatment cohort with an adjusted HR of 0.70 (95\% CI, 0.60 to 0.82; p<0.001) (Table 2). Other significant risk factors associated with increased overall mortality were age $\geq 60$ years, male sex, economic status level lower than $50 \%$, and CCI scores of 1 and $\geq 2$ (vs CCI score 0 ).

\section{Risk of cardiovascular disease, cerebrovascular disease, and overall cancer incidence considering $\boldsymbol{H}$. pylori treat- ment}

Table 3 shows the incidence risks of diseases related to common cause of deaths, including cancer, cardiovascular, and cerebrovascular diseases, according to $H$. pylori treatment. In the $H$. pylori treatment cohort, risk for cardiovascular disease incidence (adjusted HR [aHR], 1.13; 95\% CI, 1.03 to 1.24 ; $\mathrm{p}=0.01$ ) and overall cancer incidence (aHR, 1.14; 95\% CI, 1.01 to $1.28 ; \mathrm{p}=0.035$ ) were significantly higher when compared with

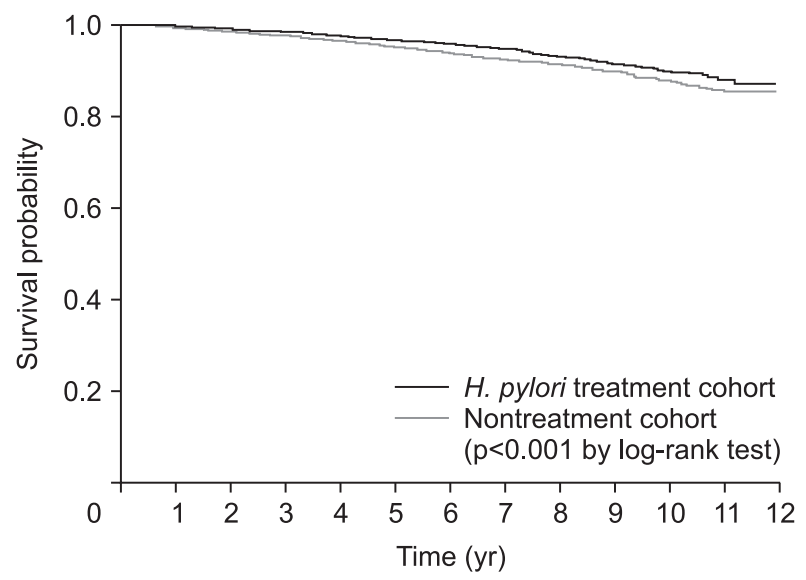

Fig. 2. Overall survival curves according to Helicobacter pylori treatment. During the follow-up period, death was reported in 229 patients (4.1\%) in the $H$. pylori treatment cohort and $608(5.5 \%)$ in the nontreatment cohort. The overall survival rate of the $H$. pylori treatment cohort was significantly higher than that of the nontreatment cohort. the nontreatment cohort. No significant difference in the risk for cerebrovascular disease incidence was found. According to cancer type, the $H$. pylori treatment cohort had significantly increased risks of colorectal cancer (aHR, 1.39; 95\% CI, 1.01 to $1.90 ; \mathrm{p}=0.043$ ) and thyroid cancer (aHR, 1.54; 95\% CI, 1.03 to $2.32 ; \mathrm{p}=0.037)$.

\section{Risk of mortalities due to cardiovascular disease, cere- brovascular disease, and overall cancer according to $\boldsymbol{H}$. pylori treatment}

The mortality risk due to cerebrovascular disease was significantly lower in the $H$. pylori treatment cohort (aHR, 0.46; 95\% CI, 0.26 to $0.81 ; p=0.007$ ) compared with the nontreatment cohort. However, mortality risk due to cardiovascular disease (aHR, 0.96; 95\% CI, 0.54 to $1.71 ; \mathrm{p}=0.896$ ) and cancer (aHR, 0.76 ; $95 \%$ CI, 0.57 to $1.01 ; \mathrm{p}=0.058$ ) were not significantly different between the $H$. pylori treatment and control cohorts. No significant association between $H$. pylori treatment and mortality risk of gastric cancer was found (aHR, 1.21; 95\% CI, 0.44 to 3.38; $\mathrm{p}=0.71$ ). However, as compared with the control group, mortality risk due to non-gastric cancers were significantly decreased (aHR, 0.73; 95\% CI, 0.55 to $0.99 ; \mathrm{p}=0.04$ ), especially liver cancer-specific mortality (aHR, 0.48; 95\% CI, 0.25 to 0.91 ; $\mathrm{p}=0.024$ ) (Table 4).

\section{DISCUSSION}

In this retrospective population-based cohort study, we investigated the effects of $H$. pylori treatment on overall mortality as well as major causes of death. We found that $H$. pylori treatment in hypertension patients was associated with a significant decrease in the risk of overall mortality and cerebrovascular disease mortality. There was no association between $H$. pylori treatment and mortality risk due to cardiovascular diseases and overall cancers.

The recent European guideline stated that $H$. pylori eradication reduces the risk of gastric cancer development. ${ }^{6}$ A metaanalysis showed that the overall mortality rate after $H$. pylori treatment was increased by $9 \%$, despite reduced gastric cancer incidence and gastric cancer mortality. ${ }^{2}$ The finding suggests that non-gastric cancer-related mortalities might be increased after $H$. pylori treatment. We also recently reported that metachronous gastric cancer risk (HR, 0.50; $p=0.03$ ) was significantly reduced in early gastric cancer patients who underwent endoscopic resection, but overall mortality risk after $H$. pylori treatment was increased (HR, 1.95; $\mathrm{p}=0.19) .{ }^{20}$ However, these studies evaluated overall mortality as a secondary outcome and found no significant differences. ${ }^{2,20}$ In our study, overall mortality was evaluated as the primary outcome, and we found that $H$. pylori treatment was not associated with increased overall mortality in patients with hypertension.

Clarithromycin is often included in several $H$. pylori treatment 
Table 2. Risk Factors Associated with Overall Mortality

\begin{tabular}{|c|c|c|c|c|c|}
\hline \multirow[b]{2}{*}{ Risk factor } & \multirow[b]{2}{*}{ No. } & \multicolumn{2}{|c|}{ Univariate analysis* } & \multicolumn{2}{|c|}{ Multivariate analysis ${ }^{*, \dagger}$} \\
\hline & & $\begin{array}{l}\text { Unadjusted HR } \\
\text { (95\% CI) }\end{array}$ & p-value & $\begin{array}{l}\text { Adjusted HR } \\
\text { (95\% CI) }\end{array}$ & p-value \\
\hline Age, yr & & & $<0.001$ & & $<0.001$ \\
\hline$<60$ & 11,877 & 1.00 & & 1.00 & \\
\hline$\geq 60$ & 4,746 & $3.99(3.46-4.59)$ & & $4.02(3.05-4.64)$ & \\
\hline Sex & & & $<0.001$ & & $<0.001$ \\
\hline Female & 9,177 & 1.00 & & 1.00 & \\
\hline Male & 7,446 & $1.60(1.39-1.84)$ & & $1.75(1.52-2.02)$ & \\
\hline Economic status level, \% & & & $<0.001$ & & $<0.001$ \\
\hline $0-50$ & 6,512 & 1.00 & & 1.00 & \\
\hline $51-100$ & 10,111 & $0.75(0.66-0.86)$ & & $0.76(0.66-0.87)$ & \\
\hline Residential area & & & 0.003 & & 0.064 \\
\hline Metropolitan & 7,925 & 1.00 & & 1.00 & \\
\hline Small city or rural & 8,698 & $1.23(1.07-1.41)$ & & $1.14(0.99-1.31)$ & \\
\hline \multicolumn{6}{|l|}{ CCI score } \\
\hline 0 & 8,658 & 1.00 & & 1.00 & \\
\hline 1 & 2,952 & $1.37(1.13-1.66)$ & 0.001 & $1.27(1.04-1.54)$ & 0.018 \\
\hline$\geq 2$ & 5,040 & $1.82(1.56-2.11)$ & $<0.001$ & $1.80(1.55-2.10)$ & $<0.001$ \\
\hline H. pylori treatment & & & $<0.001$ & & $<0.001$ \\
\hline No & 11,082 & 1.00 & & 1.00 & \\
\hline Yes & 5,541 & $0.74(0.64-0.86)$ & & $0.70(0.60-0.82)$ & \\
\hline Aspirin use & & & 0.922 & & 0.16 \\
\hline No & 13,250 & 1.00 & & 1.00 & \\
\hline Yes & 3,373 & $1.01(0.85-1.19)$ & & $0.88(0.75-1.05)$ & \\
\hline Statin use & & & 0.094 & & 0.097 \\
\hline No & 13,947 & 1.00 & & 1.00 & \\
\hline Yes & 2,676 & $0.83(0.67-1.03)$ & & $0.83(0.66-1.03)$ & \\
\hline
\end{tabular}

HR, hazard ratio; CI, confidence interval; CCI, Charlson comorbidity index; H. pylori, Helicobacter pylori.

*Analyses were performed using the Cox proportional hazard model; ${ }^{\dagger}$ Covariates for multivariate analysis were age, sex, economic status, residential area, CCI score, $H$. pylori treatment, and aspirin and statin use.

regimens, such as PPI-clarithromycin-containing triple therapy, concomitant therapy, and sequential therapy, which are recommended as the first-line treatment regimens. ${ }^{6,18,21}$ Clarithromycin has been reported to be associated with increased cardiovascular mortality risk, especially in the short-term periods after the start of the drugs, because of arrhythmia-related cardiovascular events. ${ }^{22,23}$ In addition, a prospective randomized trial reported that 2 weeks' clarithromycin treatment significantly increased long-term overall mortality until 10-year follow-up. ${ }^{7,824}$ However, the mechanisms of the increased long-term mortality risk after clarithromycin use are not clearly elucidated. In our study, however, overall mortality and cardiovascular disease mortality were not increased, even though most patients (96.4\%) in the $H$. pylori treatment cohort received a PPI-clarithromycincontaining triple therapy.

Recent retrospective cohort studies reported inconsistent results for the association between clarithromycin-containing H. pylori treatment and overall mortality. ${ }^{25-27}$ Mosholder et al. ${ }^{25}$ showed that overall mortality was increased during a median follow-up of 3.1 years after clarithromycin-containing $H$. pylori treatment when compared with the metronidazole regimen (aHR, 1.09; 95\% CI, 1.00 to 1.18). In contrast, Root et al. ${ }^{26}$ reported that overall mortality (adjusted incidence risk rate [aIRR], 0.97; $\mathrm{p}=0.66$ ) and cardiovascular mortality ( $\mathrm{IIRR}, 0.93 ; \mathrm{p}=0.69$ ) were not increased in the 3 years after $H$. pylori treatments. Interestingly, Andersen et al. ${ }^{27}$ reported that patients with ischemic heart disease who received $H$. pylori treatment had an increased risk of overall mortality for 1 year (HR, 1.50; $\mathrm{p}<0.0001)$, but no increase in the overall mortality risk $(\mathrm{HR}, 1.02 ; \mathrm{p}=0.87)$ during 5-year periods. These studies included patients who had cardiovascular diseases (coronary heart disease/ischemic heart disease, heart failure/cardiomyopathy, or arrhythmia) at baseline, which 
Table 3. Risk of Incidence for Cardiovascular Diseases, Cerebrovascular Diseases, and Cancer Stratified by $H$. pylori Treatment

\begin{tabular}{|c|c|c|c|c|c|c|}
\hline & \multicolumn{2}{|c|}{ Incidence, no. (\%) } & \multicolumn{4}{|c|}{ Risk of incidence for $H$. pylori treatment cohort* } \\
\hline & $\begin{array}{l}\text { H. pylori treatment } \\
\text { group }(n=5,541)\end{array}$ & $\begin{array}{l}\text { Control group } \\
(n=11,082)\end{array}$ & $\begin{array}{l}\text { Unadjusted HR } \\
(95 \% \mathrm{CI})\end{array}$ & p-value & $\begin{array}{l}\text { Adjusted HR } \\
\text { (95\% CI) }\end{array}$ & p-value \\
\hline Cardiovascular disease & 714 (12.9) & $1,232(11.1)$ & $1.18(1.07-1.29)$ & $<0.001$ & $1.13(1.03-1.24)$ & 0.01 \\
\hline Cerebrovascular disease & $408(7.4)$ & $814(7.3)$ & $1.00(0.89-1.13)$ & 0.962 & $0.98(0.87-1.11)$ & 0.657 \\
\hline Overall cancers & $429(7.7)$ & $737(6.7)$ & $1.18(1.04-1.32)$ & 0.008 & $1.14(1.01-1.28)$ & 0.035 \\
\hline Stomach cancer & $54(1.0)$ & $87(0.8)$ & $1.24(0.89-1.75)$ & 0.209 & $1.26(0.90-1.78)$ & 0.182 \\
\hline Non-stomach cancer & $381(6.9)$ & $659(6.0)$ & $1.17(1.03-1.32)$ & 0.017 & $1.12(0.99-1.27)$ & 0.078 \\
\hline Oral cancer & $15(0.3)$ & $19(0.2)$ & $1.58(0.80-3.11)$ & 0.185 & $1.60(0.81-3.18)$ & 0.175 \\
\hline Esophageal cancer & $3(0.1)$ & $7(0.1)$ & $0.86(0.22-3.31)$ & 0.823 & $0.82(0.21-3.20)$ & 0.771 \\
\hline Colorectal cancer & $67(1.2)$ & $95(0.9)$ & $1.42(1.04-1.93)$ & 0.03 & $1.39(1.01-1.90)$ & 0.043 \\
\hline Liver cancer & $48(0.9)$ & $101(0.9)$ & $0.95(0.67-1.34)$ & 0.774 & $0.83(0.59-1.18)$ & 0.295 \\
\hline Biliary tract cancer & $5(0.1)$ & $17(0.2)$ & $0.59(0.22-1.59)$ & 0.297 & $0.59(0.22-1.61)$ & 0.302 \\
\hline Pancreatic cancer & $21(0.4)$ & $31(0.3)$ & $1.36(0.78-2.36)$ & 0.282 & $1.25(0.71-2.19)$ & 0.435 \\
\hline Lung cancer & $34(0.6)$ & $67(0.6)$ & $1.02(0.67-1.53)$ & 0.942 & 0.99 (0.65-1.49) & 0.942 \\
\hline Breast cancer & $12(0.2)$ & $24(0.2)$ & $1.00(0.50-2.00)$ & 1.000 & $0.98(0.49-1.97)$ & 0.956 \\
\hline Prostate cancer & $58(1.0)$ & $101(0.9)$ & $1.15(0.83-1.59)$ & 0.397 & $1.13(0.81-1.56)$ & 0.472 \\
\hline Bladder cancer & $6(0.1)$ & $17(0.2)$ & $0.71(0.28-1.79)$ & 0.462 & $0.70(0.27-1.79)$ & 0.456 \\
\hline Thyroid cancer & $42(0.7)$ & $53(0.5)$ & $1.59(1.06-2.38)$ & 0.025 & $1.54(1.03-2.32)$ & 0.037 \\
\hline Brain, CNS cancer & $3(0.1)$ & $6(0.1)$ & $1.00(0.25-4.00)$ & 1.000 & $0.87(0.22-3.50)$ & 0.841 \\
\hline Non-Hodgkin lymphoma & $3(0.1)$ & $4(0.04)$ & $1.50(0.34-6.70)$ & 0.595 & $1.39(0.31-6.30)$ & 0.667 \\
\hline Leukemia & $5(0.1)$ & $3(0.03)$ & $3.34(0.80-13.97)$ & 0.099 & $3.51(0.83-14.89)$ & 0.089 \\
\hline
\end{tabular}

H. pylori, Helicobacter pylori; HR, hazard ratio; CI, confidence interval; CNS, cerebral nervous system.

*HR obtained using the Cox proportional hazard model. In the multivariate analyses, the covariates included were age, sex, economic status, residential area, Charlson comorbidity index score, and aspirin and statin use.

might have affected cardiovascular mortality risk. ${ }^{25-27}$ Gastroenterologists may be reluctant to prescribe a clarithromycincontaining $H$. pylori treatment for patients with cardiovascular diseases. To avoid selection bias and short-term cardiovascular effects of clarithromycin, we excluded patients with cardiovascular diseases other than hypertension before baseline and up to 6 months after $H$. pylori treatment.

Antibiotic treatment can cause dysbiosis and may alter the beneficial roles of gut microbiota in the modulation of carcinogenesis, response to cancer treatment including chemotherapy, immunotherapy and radiotherapy, and treatment-related toxicities. ${ }^{10,11}$ Repeated macrolide antibiotic exposure was significantly associated with increased risks of lung, gastric, biliary, and kidney cancer. ${ }^{12}$ Our recent randomized trial also showed that the $H$. pylori treatment group had a nonsignificantly higher risk of non-gastric cancer death compared with the placebo group (3.1\% vs $0.5 \%$, respectively; $\mathrm{p}=0.06$ ). ${ }^{20}$ In the current study, $H$. pylori treatment was significantly associated with overall cancer incidence risk (aHR, 1.14; $p=0.035$ ), especially colorectal cancer and thyroid cancer incidences. However, mortality risk due to overall cancer and non-gastric cancer did not increase after $H$. pylori treatment. We speculated the finding that increased overall cancer incidence was not accompanied by a corresponding mortality increase after $H$. pylori treatment might be explained by the bias associated with health behavior difference between cohorts. Patients in the treatment cohort had higher proportions of metropolitan residency, better economic status levels, and tended to use statin. In addition, some portions of patients ( 5\%) received $H$. pylori treatment without peptic ulcer disease and these patients might be treated for prophylactic eradication. Thus, the $H$. pylori treatment cohort might be more likely to attend a health examination or cancer screening program. This is supported by the finding that the incidences of colorectal cancer and thyroid cancer were both significantly increased, and both are well-known for screening-related incidence increase. ${ }^{28,29}$ However, we could not evaluate whether the stages of diagnosed colon cancer or thyroid cancer were affected according to the health behavior differences because the database did not provide cancer stage information.

Two recent meta-analyses reported inconsistent results regarding the association between $H$. pylori infection status and cerebrovascular disease risks. $^{30,31}$ A meta-analysis of casecontrol studies showed a significant association between $H$. pylori infection status and increased risk of ischemic stroke, ${ }^{30}$ but the meta-analysis of prospective cohort studies reported that $H$. pylori infection status did not increase stroke risk. ${ }^{31}$ Meanwhile, 
Table 4. Risk of Cause-Specific Mortality Stratified by H. pylori Treatment

\begin{tabular}{|c|c|c|c|c|c|c|}
\hline & \multicolumn{2}{|c|}{ Death, No. (\%) } & \multicolumn{4}{|c|}{ Risk of mortality for $H$. pylori treatment cohort* } \\
\hline & $\begin{array}{l}\text { H. pylori treatment } \\
\text { group }(n=5,541)\end{array}$ & $\begin{array}{l}\text { Control group } \\
(\mathrm{n}=11,082)\end{array}$ & $\begin{array}{l}\text { Unadjusted HR } \\
\text { (95\% CI) }\end{array}$ & $\mathrm{p}$-value & $\begin{array}{l}\text { Adjusted HR } \\
(95 \% \mathrm{CI})\end{array}$ & p-value \\
\hline Overall & $229(4.13)$ & $608(5.49)$ & & & & \\
\hline Cardiovascular disease & $18(0.32)$ & $34(0.31)$ & $1.06(0.60-1.88)$ & 0.842 & $0.96(0.54-1.71)$ & 0.896 \\
\hline Cerebrovascular disease & $15(0.27)$ & $66(0.60)$ & $0.45(0.26-0.79)$ & 0.006 & $0.46(0.26-0.81)$ & 0.007 \\
\hline Overall cancers & $68(1.23)$ & $170(1.53)$ & $0.80(0.60-1.06)$ & 0.112 & $0.76(0.57-1.01)$ & 0.058 \\
\hline Stomach cancer & $6(0.11)$ & $10(0.09)$ & $1.20(0.44-3.30)$ & 0.724 & $1.21(0.44-3.38)$ & 0.71 \\
\hline Non-stomach cancer & $62(1.12)$ & $160(1.44)$ & $0.77(0.58-1.03)$ & 0.083 & $0.73(0.55-0.99)$ & 0.04 \\
\hline Oral cancer & 0 & $2(0.02)$ & NA & & NA & \\
\hline Esophageal cancer & $2(0.04)$ & $3(0.03)$ & NA & & NA & \\
\hline Colorectal cancer & $5(0.09)$ & $17(0.15)$ & $0.59(0.22-1.59)$ & 0.297 & $0.60(0.22-1.64)$ & 0.319 \\
\hline Liver cancer & $12(0.22)$ & $45(0.41)$ & $0.53(0.28-1.01)$ & 0.052 & $0.48(0.25-0.91)$ & 0.024 \\
\hline Biliary tract cancer & $5(0.09)$ & $13(0.12)$ & $0.77(0.27-2.16)$ & 0.618 & $0.79(0.28-2.22)$ & 0.65 \\
\hline Pancreatic cancer & $5(0.09)$ & $13(0.12)$ & $0.77(0.27-2.16)$ & 0.618 & $0.67(0.24-1.91)$ & 0.456 \\
\hline Lung cancer & $15(0.27)$ & $32(0.29)$ & $0.94(0.51-1.73)$ & 0.836 & $0.95(0.51-1.75)$ & 0.857 \\
\hline Breast cancer & 0 & $1(0.01)$ & NA & & NA & \\
\hline Prostate cancer & 0 & $4(0.04)$ & NA & & NA & \\
\hline Bladder cancer & 0 & $7(0.06)$ & NA & & NA & \\
\hline Thyroid cancer & $1(0.02)$ & 0 & NA & & NA & \\
\hline Brain, CNS cancer & 0 & $1(0.01)$ & NA & & NA & \\
\hline Non-Hodgkin lymphoma & $1(0.02)$ & $3(0.03)$ & NA & & NA & \\
\hline Leukemia & $3(0.05)$ & $1(0.01)$ & NA & & NA & \\
\hline
\end{tabular}

H. pylori, Helicobacter pylori; HR, hazard ratio; CI, confidence interval; CNS, cerebral nervous system; NA, not available.

*HR obtained using the Cox proportional hazard model. In the multivariate analyses, the covariates included were age, sex, economic status, residential area, Charlson comorbidity index score, and aspirin and statin use.

in a recent population-based study, subgroup analysis including patients who received $H$. pylori eradication showed that stroke risk was not increased after the clarithromycin-containing $H$. pylori treatment. ${ }^{23}$ Similarly, we found that cerebrovascular disease risk was not increased after $H$. pylori treatment (aHR, 0.98; $\mathrm{p}=0.657$ ). However, mortality risk due to cerebrovascular disease was significantly decreased by $54 \%$ after $H$. pylori treatment. The reason for the lower cerebrovascular mortality risk in the $H$. pylori treatment group remains unclear.

Our study has several limitations associated with an observational study of a retrospective cohort study design. First, our study population was hypertension patients and our results might not be applicable to the general population with no cardiovascular risk. Second, the ICD-10 disease codes for hypertension were used for selection of the study population from the database, and possible upcoding for hypertension diagnosis could not be completely excluded. However, to reduce the possibility, we only selected patients who had a prescription for an anti-hypertensive drug. Third, our analysis was based on the prescription of $H$. pylori treatment regimen. Neither actual compliance with the prescribed drugs nor $H$. pylori status after treatment was available from the database. Also, the nontreatment cohort included patients who might be infected but not treated and those who were not infected. Fourth, the use of antithrombotic agents or antiplatelet agents other than aspirin which might affect the study outcomes was not included, because proportions of preventive use of such agents other than aspirin or statin might be low in our study patients who had not been previously diagnosed with cardiovascular diseases or cerebrovascular diseases before $H$. pylori treatment. Fifth, the proportion of patients who received only bismuth-containing quadruple therapy was $1.3 \%$, and majority of the study outcomes might derive from the effects of PPI-clarithromycin containing triple therapy. Finally, lifestyle factor variables present at baseline such as smoking, alcohol, or body mass index that might affect incidence and mortality of our study outcome diseases were frequently missing from the available dataset.

In conclusion, $H$. pylori treatment in patients with hypertension was not significantly associated with increased overall mortality. When analyzed by the major causes of death, $H$. pylori treatment did not increase mortalities due to cardiovascular diseases, cerebrovascular diseases, or overall cancers. Further prospective studies are needed to confirm these findings before implementing a policy of $H$. pylori eradication for the purpose 
of gastric cancer prevention in general populations.

\section{CONFLICTS OF INTEREST}

No potential conflict of interest relevant to this article was reported.

\section{ACKNOWLEDGEMENTS}

This research was supported by a grant from the Korea Health Technology R\&D Project through the Korea Health Industry Development Institute (KHIDI), funded by the Ministry of Health \& Welfare, Republic of Korea (grant number: HC15C1077). This study used the Korean National Health Insurance ServiceNational Sample Cohort database (NHIS-2016-2-191) provided by the National Health Insurance Service.

\section{AUTHOR CONTRIBUTIONS}

Interpretation of the data: Y.I.K., Y.A.K., I.J.C. Drafting of the article: Y.I.K., I.J.C. Statistical analysis: Y.A.K., J.W.L. Conception and design of study: I.J.C. Critical revision for important intellectual content and final approval of the manuscript: Y.I.K., Y.A.K., J.W.L., H.J.K., S.H.K., S.G.K., J.I.K., J.J.K., I.J.C.

\section{ORCID}

Young-Il Kim

Young Ae Kim

Jang Won Lee

Hak Jin Kim

Su-Hyun Kim

Sang Gyun Kim

Jin Il Kim

Jae J. Kim

Il Ju Choi https://orcid.org/0000-0002-2614-3419
https://orcid.org/0000-0002-3819-0028
https://orcid.org/0000-0001-9208-1829
https://orcid.org/0000-0003-2483-1469
https://orcid.org/0000-0002-0679-0918
https://orcid.org/0000-0003-1799-9028
https://orcid.org/0000-0001-6801-6891
https://orcid.org/0000-0002-0226-1330
https://orcid.org/0000-0002-8339-9824

\section{REFERENCES}

1. Schistosomes, liver flukes and Helicobacter pylori. IARC Working Group on the Evaluation of Carcinogenic Risks to Humans. Lyon, 7-14 June 1994. IARC Monogr Eval Carcinog Risks Hum 1994;61:1-241.

2. Ford AC, Forman D, Hunt RH, Yuan Y, Moayyedi P. Helicobacter pylori eradication therapy to prevent gastric cancer in healthy asymptomatic infected individuals: systematic review and metaanalysis of randomised controlled trials. BMJ 2014;348:g3174.

3. Statistics Korea. Statistics for cause of death [Internet]. Daejeon: Statistics Korea [cited 2018 Jul 30]. Available from: http://kostat. go.kr/portal/korea/kor_nw/1/1/index.board.

4. World Health Organization (WHO). The top 10 causes of death [Internet]. Geneva: WHO; c2018 [cited 2018 Aug 17]. Available from: http://www.who.int/news-room/fact-sheets/detail/the-top10-causes-of-death

5. China Statistics Press. China Statistical Yearbook 2016 [Internet]. Beijing: National Bureau of Statistics [cited 2018 Aug 17]. Available from: http://www.stats.gov.cn/tjsj/ndsj/2016/indexeh.htm.

6. Malfertheiner P, Megraud F, O'Morain CA, et al. Management of Helicobacter pylori infection: the Maastricht V/Florence consensus report. Gut 2017;66:6-30.

7. Jespersen CM, Als-Nielsen B, Damgaard M, et al. Randomised placebo controlled multicentre trial to assess short term clarithromycin for patients with stable coronary heart disease: CLARICOR trial. BMJ 2006;332:22-27.

8. Gluud C, Als-Nielsen B, Damgaard M, et al. Clarithromycin for 2 weeks for stable coronary heart disease: 6-year follow-up of the CLARICOR randomized trial and updated meta-analysis of antibiotics for coronary heart disease. Cardiology 2008;111:280-287.

9. Cheng YJ, Nie XY, Chen XM, et al. The role of macrolide antibiotics in increasing cardiovascular risk. J Am Coll Cardiol 2015;66:2173-2184.

10. Dzutsev A, Goldszmid RS, Viaud S, Zitvogel L, Trinchieri G. The role of the microbiota in inflammation, carcinogenesis, and cancer therapy. Eur J Immunol 2015;45:17-31.

11. Roy S, Trinchieri G. Microbiota: a key orchestrator of cancer therapy. Nat Rev Cancer 2017;17:271-285.

12. Boursi B, Mamtani R, Haynes K, Yang YX. Recurrent antibiotic exposure may promote cancer formation: another step in understanding the role of the human microbiota? Eur J Cancer 2015;51:2655-2664.

13. Taler SJ. Initial treatment of hypertension. N Engl J Med 2018;378:636-644.

14. Lee J, Lee JS, Park SH, Shin SA, Kim K. Cohort profile: the National Health Insurance Service-National Sample Cohort (NHISNSC), South Korea. Int J Epidemiol 2017;46:e15.

15. Lee SS, Ae Kong K, Kim D, et al. Clinical implication of an impaired fasting glucose and prehypertension related to new onset atrial fibrillation in a healthy Asian population without underlying disease: a nationwide cohort study in Korea. Eur Heart J 2017;38:2599-2607.

16. Kang YM, Kim YJ, Park JY, Lee WJ, Jung CH. Mortality and causes of death in a national sample of type 2 diabetic patients in Korea from 2002 to 2013. Cardiovasc Diabetol 2016;15:131.

17. World Health Organization (WHO). ICD-10: International statistical classification of diseases and related health problems. 10th ed. Geneva: WHO, 1992.

18. Kim SG, Jung HK, Lee HL, et al. Guidelines for the diagnosis and treatment of Helicobacter pylori infection in Korea, 2013 revised edition. J Gastroenterol Hepatol 2014;29:1371-1386.

19. Quan H, Li B, Couris CM, et al. Updating and validating the Charlson comorbidity index and score for risk adjustment in hospital discharge abstracts using data from 6 countries. Am J Epidemiol 2011;173:676-682.

20. Choi IJ, Kook MC, Kim YI, et al. Helicobacter pylori therapy for 
the prevention of metachronous gastric cancer. N Engl J Med 2018;378:1085-1095.

21. Chey WD, Leontiadis GI, Howden CW, Moss SF. ACG clinical guideline: treatment of helicobacter pylori infection. Am J Gastroenterol 2017;112:212-239.

22. Svanström H, Pasternak B, Hviid A. Use of clarithromycin and roxithromycin and risk of cardiac death: cohort study. BMJ 2014;349:g4930.

23. Wong AY, Root A, Douglas IJ, et al. Cardiovascular outcomes associated with use of clarithromycin: population based study. BMJ 2016;352:h6926.

24. Winkel P, Hilden J, Hansen JF, et al. Clarithromycin for stable coronary heart disease increases all-cause and cardiovascular mortality and cerebrovascular morbidity over 10years in the CLARICOR randomised, blinded clinical trial. Int J Cardiol 2015;182:459-465.

25. Mosholder AD, Lee JY, Zhou EH, et al. Long-term risk of acute myocardial infarction, stroke, and death with outpatient use of clarithromycin: a retrospective cohort study. Am J Epidemiol 2018;187:786-792.
26. Root AA, Wong AY, Ghebremichael-Weldeselassie Y, et al. Evaluation of the risk of cardiovascular events with clarithromycin using both propensity score and self-controlled study designs. Br J Clin Pharmacol 2016;82:512-521.

27. Andersen SS, Hansen ML, Norgaard ML, et al. Clarithromycin use and risk of death in patients with ischemic heart disease. Cardiology 2010;116:89-97.

28. Ahn HS, Kim HJ, Welch HG. Korea's thyroid-cancer "epidemic": screening and overdiagnosis. N Engl J Med 2014;371:1765-1767.

29. Shin A, Kim KZ, Jung KW, et al. Increasing trend of colorectal cancer incidence in Korea, 1999-2009. Cancer Res Treat 2012;44:219-226.

30. Wang ZW, Li Y, Huang LY, et al. Helicobacter pylori infection contributes to high risk of ischemic stroke: evidence from a metaanalysis. J Neurol 2012;259:2527-2537.

31. Yu M, Zhang Y, Yang Z, Ding J, Xie C, Lu N. Association between Helicobacter pylori infection and stroke: a meta-analysis of prospective observational studies. J Stroke Cerebrovasc Dis 2014;23:2233-2239. 